\title{
Il dialetto napoletano si deve scrivere come si parla? Polemiche ottocentesche sull'ortografia del napoletano
}

\section{Gabriella Gavagnin}

Universitat de Barcelona

\begin{abstract}
L'articolo ricostruisce le diverse fasi di una polemica linguistica ottocentesca fra promotori di una riforma ortografica del napoletano basata sul parlato contemporaneo e difensori della norma grafica derivata dai classici della tradizione dialettale scritta. Si cerca inoltre di individuare le ragioni extralinguistiche che influenzano le posizioni dei diversi schieramenti e le implicazioni fra tale dibattito e le nuove prospettive che si aprono per l'italiano e i dialetti all'indomani dell'unificazione politica.
\end{abstract}

Parole chiave: storia linguistica del napoletano, dialetto scritto e dialetto parlato, Vittorio Imbriani.

\begin{abstract}
The article reconstructs the diverse phases of a $19^{\text {th }}$ century linguistic controversy between the promoters of an orthographic reformation of Napolitan based on contemporary speech, and the defenders of the rules of writing, derived from the classics, pertaining to the tradition of written dialect. It also attempts to individualise the extra-linguistic reasons that influence the position of the many and varied groups opposed to one another, as well as the implications of this debate and the new perspective that it opened up both for Italian and for the dialects, once political unification had taken place.
\end{abstract}

Key words: the linguistic history of Napolitan, written and spoken dialect, Vittorio Imbriani

Nella seconda metà dell'Ottocento, a distanza di un secolo dalle riflessioni e dalle polemiche provocate dal trattatello storico-linguistico di Ferdinando Galiani, nuovi e svariati fattori concorsero alla nascita di un dibattito nella cultura napoletana sull'opportunità e sui modi di riformare e fissare una norma grafica (e linguistica) del dialetto. Gli interventi si susseguirono per un paio di decenni in prefazioni, opuscoli, discorsi accademici e, soprattutto, sulle pagine di pubblicazioni periodiche come il Giambattista Basile e il San Carlino, stimolando peraltro una crescente attività grammaticale e lessicografica che si tradusse sia in articoli eruditi che in manuali di ortografia e grammatica e in 
vocabolari bilingui. Nelle pagine che seguono cercherò di ripercorrere i momenti salienti delle discussioni che accanirono letterati e grammatici negli anni postunitari allo scopo di scorgere, dietro le concrete proposte linguistiche, le prospettive teoriche da cui muovevano le diverse posizioni e di capire fino a che punto le polemiche scaturivano da un tentativo di ridefinizione dei rapporti fra dialetto e lingua.

La questione fu sollevata, inizialmente senza spirito polemico, dall'esigenza degli studiosi di demologia di fissare una serie di norme ortografiche atte a trascrivere il patrimonio di letteratura popolare orale che venivano raccogliendo nella loro attività di ricerca sul territorio. In particolare, Vittorio Imbriani, principale propulsore di questi studi nell'area meridionale, affronta per la prima volta il problema nel dare alle stampe, nel 1871, i Canti popolari delle provincie meridionali, raccolti insieme al pugliese Antonio Casetti nel decennio precedente. ${ }^{1}$ Il sistema ortografico tramandato dalla letteratura colta gli si rivela del tutto inadeguato alla trascrizione di una serie di parlate dialettali che, oltre a presentare notevoli variazioni diatopiche, avevano ormai raggiunto, a seguito di un naturale processo evolutivo che aveva colpito tratti fonetici e morfosintattici caratterizzanti, un grado di sviluppo che le allontanava dal modello linguistico su cui si era basata la letteratura colta secentesca e settecentesca. Per questo, Imbriani stabilisce una serie di criteri decisamente innovativi, che manterrà nel complesso invariati in edizioni posteriori di altra letteratura orale, malgrado i dissensi e le aspre critiche che la sua riforma scatenò. Occorre notare che nella scelta dei criteri di trascrizione pesarono due fattori strettamente correlati: da una parte, la volontà di riprodurre, senza attenuazioni o ricreazioni di nessun tipo, modi ed espressioni linguistiche dell'informatore, di assumere insomma, con la fedeltà diplomatica dovuta ai documenti storici, la parlata popolare, quale voce autentica e spontanea, depositaria di una cultura antica e pregiata; dall'altra, l'intento di collocare tale cultura, tanto nella lingua quanto nei contenuti, nel quadro di una cultura sovraregionale, nazionale, come parte integrante e funzionale di essa. Quanto al primo punto, è più che esplicita la seguente dichiarazione di principio, appartenente a uno scritto del 1866:

Il seguire esattamente coll'ortografia le menome inflessioni del linguaggio parlato; il non mutare nemmanco una parola; l'indicare la patria di chi canta la poesia; il soggiungere quanti più chiarimenti si può sulle allusioni in essa contenute, son cose che già s'intendono per sé. ${ }^{2}$

1. Una visione d'insieme dell'attività di demologo d'Imbriani è data da Alberto Mario CireSE, «Imbriani demopsicologo», in R. FranZeSE e E. GIAMmatTeI (a cura di), Studi su Vittorio Imbriani. Atti del Primo Convegno su Vittorio Imbriani nel Centenario della morte, Napoli: Guida, 1990, p. 165-197; sulle implicazioni filologiche e linguistiche di tale attività, si veda, nel medesimo volume di Atti, il contributo di Patricia BIANCHI, «Imbriani editore di letteratura orale», p. 465-476.

2. V. IMBRIANI, Mucchietto di gemme, Napoli: s.t., 1866, p. 21 (cit. da BIANCHI, op. cit., p. 467). 
Quanto al secondo, va ricordato innanzitutto che, nel suo atteggiamento nei confronti della questione della lingua prevalgono, in consonanza con le tendenze marcatamente espressioniste della sua prosa narrativa, posizioni antipuriste e antimanzoniane, in una sorta di «liberismo di stampo ascoliano», ${ }^{3}$ il che riflette una visione della cultura nazionale fondata sulla forte saldatura di tutte le componenti regionali. In questa prospettiva, appare chiaro che le culture dialettali sono prive di qualsiasi autonomia e possono, anzi devono, essere valorate e recuperate solo nella loro funzione di costruzione di una più organica cultura italiana. Particolarmente significative al riguardo sono le affermazioni contenute nel saggio Dell'organismo poetico e della poesia popolare italiana, risultato delle lezioni del corso accademico 1862-1863:

Le letterature de' nostri dialetti, ricche spesso di opere meravigliose [...] non costituiscono un tutto compiuto, organico; perché l'ingegno delle singole parti d'Italia non è giunto mai ad affermarsi come qualcosa d'indipendente, come negazione della mente nazionale; e quindi gli autori che adoperano il dialetto sono [...] Italiani in fondo all'anima.

La militanza patriottica di queste dichiarazioni, per quanto esse arrivino in un particolare clima politico, quello degli anni immediatamente successivi all'unificazione, definisce senza equivoci il ruolo vicario assegnato alla letteratura dialettale sia nel presente che nel passato. In coerenza con tale posizione, Imbriani non a caso sottolinea, fra le novità adottate nel suo sistema di trascrizione, l'uso dell'apostrofo con valore diacritico e, soprattutto, come indicatore dello statuto subalterno del dialetto nei confronti dell'italiano. La motivazione, già addotta nell'avvertenza al primo volume dei Canti popolari meridiona$l i$, è ribadita senza titubanze nella prefazione ai XII Conti pomiglianesi del 1876:

Considerando quindi ogni vocabolo vernacolo come alterazione della voce etimologicamente corrispondente nello Italiano aulico, indico con un apostrofo ogni aferesi ed ogni apocope, ancorché il vocabolo nel dialetto esista solo in quella forma apocopata od aferizzata. Mi sembra, che, in tal modo, ne sia facilitata l'intelligenza al lettore e si ottenga di distinguere parole, che suonano press'a poco identicamente, sebbene diversissime di significato, esempligrazia 'no (uno, articolo indeterminato) no (no) e no' (non); 'sse (queste) e sse (sè); stà' (questa) e stà' (stare) eccetera.

Il sistema grafico di Imbriani è pensato per un dialetto e non per una lingua: quindi si propone anche di evidenziare la distanza fra dialetto e lingua, allo scopo (niente affatto scontato) ${ }^{4}$ di renderlo più trasparente al lettore avvez-

3. Luca Serianni, «La lingua di Vittorio Imbriani», in R. FranZeSE e E. Giammattei (a cura di), Studi su Vittorio Imbriani, cit., p. 40.

4. Così contestava le ragioni di Imbriani il giovane demologo irpino Giulio Capone, editore di XL Canti popolari inediti di Montella (Napoli: Giannini, 1881): «L'uso degli apostrofi poggia tutto su un principio etimologico molto discutibile. Glottologicamente parlando, non è giusto far rimontare la forma vernacola alla forma aulica, perché questa non è pree- 
zo all'italiano. Di qui nasce l'assunzione di un consistente apparato di apostrofi e di accenti. In questo, come su altri aspetti, lo segue il suo discepolo Gaetano Amalfi, il quale, anzi, ne accentua maggiormente l'uso. ${ }^{5}$

Una delle prime risposte polemiche alla proposta di Imbriani è contenuta nel Vocabolario di Raffaele D'Ambra. ${ }^{6}$ Nella prefazione, dopo aver asserito che "la lingua del popolo napolitano", che il vocabolario si propone di contribuire a standardizzare, "poco varia dalla lingua del popolo toscano, salvo alcuni pochi accidenti regionali che si voglion dire etnici» (il che vale a dire implicitamente che essa può vantare un'analoga dignità di lingua), D'Ambra esprime il suo netto dissenso nei confronti di coloro che per «l'esagerazione di significar graficamente il suono» nonché per «una certa frenesia di render singolare il dialetto", sono stati spinti a "raddoppiar consonanti, a scambiarle tra loro, a moltiplicare le forme dell'aferesi, dell'apocope, della paragoge, a costrignere e contorcere parole comuni e forestiere, allontanandosi dalla sincera e genuina scrittura de' lor progenitori». L'insofferenza mostrata davanti alla riforma ortografica di Imbriani e dei suoi seguaci deriva dal fatto che in essa è individuata una sorta di degradazione del napoletano, nella misura in cui sancisce come unica realtà linguistica possibile quella del parlato popolare, accogliendo tutti i fenomeni evolutivi più recenti nella loro multiforme variazione diatopica e diastratica. Non a caso, gli preme ricordare che se si scrivessero anche «le lingue note» così come "s'ode a pronunziare, ne tornerebbero lingue da selvaggi, irte, fere e bestiali». Era questa, dunque, l'immagine del napoletano che la lettura dei canti popolari, trascritti appunto con uno spirito di estremo rispetto filologico verso il parlato e le sue oscillazioni, poteva suscitare.

La risposta di Imbriani non si fa attendere molto. Nei XII Conti pomiglianesi del 1876, glossa la distinzione che D’Ambra aveva fatto a proposito delle due varianti dell'articolo ( $\mathrm{L}^{\prime} a$, in luogo dell'articolo $l a$, si tollera nel dialetto parlato; ma è un errore nella scrittura, dove si ha a segnare tutto intero») con una squalifica dell'argomentazione di fondo che non lascia alcuno spiraglio a repliche:

$\mathrm{Ci}$ avevamo la lingua scritta e la parlata: ora, il D'Ambra ci vuol regalare anche il dialetto scritto ed il parlato, tanto per aumentar la confusione. Questo dia-

sistente, ma coesistente, anzi in molti casi posteriore. L'intelligenza della parola vernacola non è gran che facilitata dall'apostrofo; e lo stesso fine si raggiunge con note, lessici, traduzioni ecc. Quanto alla pronunzia, è chiaro che l'apostrofo non l'agevola, non fa anzi che imbrogliarla. Si aggiunga inoltre, che l'uso di quel benedetto apostrofo è spesso incerto, dipendendo dal criterio etimologico dello scrittore» («L'ortografia del dialetto napoletano», Giambattista Basile, II, n. 5, 15 maggio 1884, p. 1).

5. La sua proposta (riassunta in G. AMALFI, «L'ortografia del dialetto napoletano», Giambattista Basile, II, n. 1, 15 gennaio 1884, p. 3-5), oltre ad affrontare i problemi relativi a raddoppiamenti consonantici iniziali, aferesi e apocopi, già ristrutturati da Imbriani, avanza anche un'ipotesi di trascrizione della vocale neutra finale consistente in una $e$ con un puntino sopra.

6. Vocabolario napolitano-toscano domestico di arti e mestieri, Napoli: ed. a spese dell'autore, 1873 (rist. anastatica Bologna: Forni, 1969). 
letto scritto, diverso dal parlato, non altro sarebbe se non un gergo convenzionale: e tale è pur troppo; giacché piace al più gli scrittori vernacoli di storpiar del pari la lingua aulica e lo idioma domestico.

Prima di cogliere le implicazioni di queste affermazioni, giova precisare che, nel corso di tutta la polemica che si snoderà durante l'ultimo quarto di secolo, si intrecciano due risvolti fondamentali, attorno ai quali poi sono raggruppabili una serie di disquisizioni su aspetti concreti: da un lato si dibatte sull'opportunità e sulla maniera di riformare il sistema ortografico seguendo criteri più o meno foneticisti; dall'altro, ciò che si mette in discussione è la legittimità dell'esistenza e della ricerca di uno standard. In tal senso, il caso così controverso dell'articolo riunisce emblematicamente le due facce del problema: un conto è, infatti, la disputa fra quelli che ritengono conveniente l'uso dell'apostrofo ('o, 'a) di fronte a quelli che preferirebbero prescinderne $(o, a)$, un altro conto, invece, è l'acuta e sostanziale divergenza fra i fautori della forma tradizionale $(l o, l a)$ e quelli della forma affermatasi nel parlato contemporaneo, ridotta all'unico fonema vocalico. Consapevole delle diverse implicazioni di queste posizioni, uno dei protagonisti, Giulio Capone, schieratosi fra le file dei riformatori benché critico nei confronti di Imbriani, delineò con molta approssimazione la dinamica del dibattito in uno scritto che rimase inedito e che è stato recentemente riportato alla luce:

Prima di tutto, bisogna dividere in due classi ben distinte coloro che hanno trattato dell'Ortografia napoletana: da una parte stanno i tradizionalisti i quali non si vogliono allontanare dagli scrittori classici napoletani, e pei quali esiste un dialetto scritto e un dialetto parlato; dall'altra parte ci sono i novatori, i quali stabiliscono il principio che il dialetto si deve scrivere come si parla. I tradizionalisti son tutti d'accordo, i novatori invece vanno suddivisi in più scuole, secondo che accettano o rifiutano alcuni criteri ortografici. ${ }^{7}$

Se effettivamente il conflitto più profondo consisteva nel definire il rapporto fra scritto e parlato, dalle dichiarazioni finora riportate appare già con evidenza che tale rapporto non si pone solo in termini diacronici di scelta fra un modello arcaizzante e un modello aderente al linguaggio vivo e attuale, ma coinvolge anche altri livelli di variazione. In pratica, la contrapposizione fra tradizionalisti e novatori è il risultato di una distanza ideologica che comporta anche una diversa concezione del dialetto e della sua funzione storica e sociale. Tornando alla chiosa di Imbriani a D’Ambra citata più sopra, possiamo dire che essa deriva da una visione del dialetto diastraticamente monolitica: al dialetto è negata qualsiasi escursione di registro verso l'alto, non solo nella sua

7. G. CAPONE, Scritti inediti sui dialetti napoletano e irpino, introduzione di Antonio Brescia, Avellino: Edizioni La Ginestra, 1997, p. 31. Si tratta della versione integra dell'articolo pubblicato sul Giambattista Basile nel 1884 che ho citato in una nota precedente. La parte rimasta inedita si sofferma a controbattere le posizioni di coloro che difendevano il modello linguistico della tradizione scritta. 
prospettiva presente e futura, quanto addirittura nella sua realtà storica. La lingua usata nella letteratura dialettale (e si badi che ci si riferisce a una tradizione letteraria imponente, che nel Seicento era stata capace di articolarsi nei tre generi della poesia, della prosa e del teatro) non è riconducibile a un unico sistema linguistico che comprenda la varietà parlata. Quella lingua, il dialetto scritto da Basile e da Cortese è, alla stregua di un linguaggio maccaronico, una contaminazione fra dialetto e italiano, e pertanto una corruzione di entrambi. L'idea è ripresa ripetutamente in diversi interventi pubblicati sulla principale piattaforma dei demologi, il Giambattista Basile, rivista dedicata agli studi sulla letteratura popolare e diretta da Luigi Molinaro del Chiaro. Giulio Capone vi scriveva: "con quale dritto si può parlare di un dialetto scritto e "polito" contrapposto a un dialetto parlato e "rude", mentre a Napoli non esiste che un dialetto solo, parlato dal popolo e alterato dagli scrittori?», ${ }^{8}$ mentre Gaetano Amalfi sottolinea il carattere eccentrico della letteratura dialettale barocca degli scrittori napoletani, nata da un "peculiare loro ghiribizzo», e lamenta che, poiché si trovavano in una posizione "psicologicamente ingiustificabile» ed essendo «impossibil ripetere l'ingenuità del popolo», preferirono, a torto, «trasformare il dialetto", sicché "ne uscì un sermone mescidato». ${ }^{9}$ Ancora, quando Vincenzo Arabia, Raffaele Della Campa e Guglielmo Méry si accingono a ricostruire a caldo le diverse posizioni e proposte in una serie di articoli pubblicati nel 1887 sulla medesima rivista e poi raccolti nel volume L'ortografia del dialetto napolitano, si adoperano fra l'altro per censire stranierismi, italianismi e neologismi presenti nei classici napoletani allo scopo di dimostrare che non usarono "il vero e solo vernacolo napolitano» ${ }^{10}$ poiché "non scrissero la parlata della plebe di Napoli», ${ }^{11}$ per la qual cosa «non possono formar testo nè autorità in fatto di purezza e di ortografia». ${ }^{12} \mathrm{Va}$ anche detto che la fallace contrapposizione fra un dialetto vero e unico e un dialetto falso, alterato e corrotto che vediamo emergere da queste polemiche linguistiche a proposito del dialetto scritto finì per estendersi significativamente nell'ambito della critica letteraria. Essa servì per classificare, in modo riduttivo e fuorviante, due diversi usi stilistici nei maggiori scrittori contemporanei, Ferdinando Russo e Salvatore Di Giacomo, i quali si rivolgevano al dialetto partendo da due poetiche differenziate e da due diversi atteggiamenti nei confronti della realtà. Tale semplificazione è ricaduta negativamente soprattutto su Di Giacomo, la cui ricerca stilistica lo spingeva al di là dell'orizzonte mimetico del realismo bozzettistico conquistando territori inediti per la poesia in dialetto. In un saggio recente Nicola De Blasi, correggendo questi pregiudizi prevalsi a lungo nella storia della critica, ha chiarito i termini dell'equivoco:

8. Ibid., p. 26.

9. G. AMALFI, «Come va scritto il vernacolo partenopeo?», Giambattista Basile, v, n. 6, 15 giugno 1887 , p. 41-45, citazioni p. 41-42.

10. V. Arabia, R. Della Campa e G. Méry, L'ortografia del dialetto napolitano. Appunti, osservazioni e proposte, Napoli: Pierro, 1887, p. 89.

11. Ibid., p. 45.

12. Ibid., p. 44. 
La lingua digiacomiana nel confronto con quella di Russo ha rischiato di essere fraintesa, in quanto non immediatamente riconducibile all'uso popolare informale. Ma dal momento che nel dialetto, - come nella lingua - , convivono varietà e registri diversi, la differenza tra due modi di scrivere non si deve ridurre all'opposizione dialetto «vero»/ dialetto «falso», quasi che il dialetto «vero» fosse uno solo, ma deve essere valutata in un altro modo. Di fatto il distacco dal mimetismo comporta in Di Giacomo l'adozione di uno stile poetico; egli, cioè, per ricorrere a una tautologia chiarificatrice, scrivendo testi poetici in napoletano usa un napoletano letterario, che non è né "falso", né italianizzato. ${ }^{13}$

La necessità di ribadire un'idea come quest'ultima, che dovrebbe essere ovvia, si spiega in fondo perché essa è assunta come naturale solo per la letteratura in lingua, mentre per la letteratura in dialetto pesa fortemente nel corso del Novecento, specie fino a metà secolo, la tendenza a rilegarla automaticamente in ambiti non concorrenziali, come l'universo del comico e il realismo bozzettistico o documentale. Erano questi, in sintesi, gli usi letterari scritti che i riformatori degli anni postunitari erano disposti ad assumere e a riservare per il dialetto.

Tornando alle diverse fasi del dibattito linguistico, va detto che il polo intorno a cui si articola l'opposizione alla riforma è costituito dall'Accademia dei Filopatridi. Alcuni anni prima degli articoli finora citati di Amalfi e di Capone, apparsi nel 1884 sul Giambattista Basile, essa intervenne ad accendere la disputa con un libriccino, Il dialetto napolitano si deve scrivere come si parla?, dove si stampava, insieme alla delibera dell'adunanza degli accademici del 30 giugno 1878 dedicata a tale questione, i discorsi letti in quella sede dal presidente, Emmanuele Rocco, e da Giacomo Bugni, e uno scritto di Ferdinando Taglioni inerente le implicazioni che la riforma comportava nel linguaggio musicale. Un primo elemento che occorre tener presente è che l'Accademia appare estranea e riluttante nei confronti degli studi storici positivisti e delle poetiche veriste e naturaliste che avevano trasformato la vita e la cultura popolare in oggetto di studio e in materia letteraria. In rapporto a tale atteggiamento, essa esprime, sul piano più strettamente linguistico, la tradizionale reazione del classicismo di fronte alle rivendicazioni dell'uso parlato della gente incolta, applicando al napoletano principi e dinamiche linguistiche che erano serviti per combattere un determinato modello d'italiano. Nel discorso letto da Emanuele Rocco si riscontrano, per esempio, espliciti richiami alle polemiche antifiorentiniste:

Lo stesso sta succedendo pel nostro dialetto, ed alcuni già ci sono che vanno gridando doversi scrivere come si parla, e non doverci essere differenza alcuna fra dialetto parlato e dialetto scritto, ed i nostri maestri in ciò dover essere i trecconi e i barulli del Mercato, l'infima plebe del Mandracchio e del Lavinaro. ${ }^{14}$ 
È superfluo osservare che era ben lungi da Imbriani, peraltro d'ideologia tutt'altro che progressista, rinvenire nei parlanti dialettofoni alcun tipo di modello imitabile. Occorre anche dire, però, che in questo passo si svolge una polemica immediata contro $O ̈$ Mandracchio vennecato, un volumetto di versi e prose preceduti da una prefazione dal tono provocatorio, in cui l'autore, il sacerdote Gennaro Santaniello, aveva scelto (persino nel testo introduttivo) il registro più basso e popolare. ${ }^{15}$ Come riportano Arabia, Della Campa e Méry, l'irriverenza del tono e del linguaggio suscitarono «una santa indignazione» ${ }^{16}$ fra gli accademici, che si arroccarono in difesa dei valori della tradizione. Ma il discorso di Rocco e la delibera dell'Accademia sulla controversia rispondono non solo al Mandracchio, interpretato come un'ulteriore manifestazione di una pratica che si andava consolidando, ma anche a Imbriani, che finiva per essere accoppiato a Santaniello come se si trattasse di un fronte omogeneo. L'Accademia, costituitasi sul modello cruscante allo scopo di «studiare ed illustrare gli scrittori del nostro dialetto, stabilire le regole dell'ortografia e rendere morale il teatro", ${ }^{17}$ davanti alle proposte di riforma ortografica di chi considerava come unica realtà dialettale il parlato rigorosamente contemporaneo, nella sua magmatica frammentazione geografica, decide di liquidare il problema sostenendo che il modello dialettale scritto può essere concepito solo in un rapporto di continuità con la tradizione letteraria e di indipendenza dal parlato: «quantunque la plebe, parlando aferizzi gli articoli e le preposizioni, nello scrivere si debba seguir l'esempio degli autori che ci precedettero». ${ }^{18}$

Va osservato che in ambedue gli schieramenti si avverte in modo più o meno esplicito l'eco di certe affermazioni del trattatello di Galiani Del dialetto napoletano (1779), ${ }^{19}$ il precedente teorico di maggior spessore che aveva, fra le altre cose, affrontato anche gli aspetti ortografici. Sul versante dei tradizionalisti, per esempio, D’Ambra, come si osserva nell'introduzione al

venienza musicale, Napoli: Livigni, 1879, p. 11. Il discorso di Rocco (Dialetto scritto e dialetto parlato) è riprodotto alle p. 11-14, quello di Giacomo Bugni (Osservazioni sul discorso del prof. Emmanuele Rocco) alle p. 15-27.

15. Ö Mandracchio vennecato, lébretiello sfruculijatore e ncucciuso che ncènzia, pitta e ghianghéa comme ricàpita scritto c'a vera lengu'é Napole e prubbecato ògne quìnnece juorne ra G. Santaniello, Napoli: Stamparija é ll'a ccarèmia riale r'é sciénzie, ucchijata ra Michele de Rubertis, 1877.

16. V. Arabia, R. Della Campa e G. Méry, op. cit., p. 29. Va precisato che Guglielmo Méry, prima di assumere una posizione critica nei confronti dell'Accademia dei Filopatridi, ne era stato segretario, ed aveva firmato pertanto, accanto a Emmanuele Rocco, la delibera pubblicata nel libriccino del 1879.

17. Il dialetto napolitano si deve scrivere come si parla?, cit., p. 7.

18. Ibid., p. 8-9.

19. Sulla proposta linguistica di Galiani che auspica un uso scritto colto del dialetto simile a quello che il "patriotico zelo de' Veneziani sul loro niente più armonioso dialetto è riuscito di fare», e sul suo significato storico di recupero di prestigio linguistico nel quadro di una rinascita culturale del regno, si rinvia alla prefazione di Enrico Malato alla sua edizione del saggio galianeo (F. GALIANI, Del dialetto napoletano, Roma: Bulzoni, 1970, p. IXXXIII). 
suo vocabolario sopra citata, ne recupera la tesi centrale secondo la quale esiste un volgare illustre napoletano, distinto dal sermo plebeius, che poco si discosta dalla lingua italiana colta; analogamente, Rocco sostiene che la raffinatezza che distingue lo scritto dal parlato "fu dai classici scrittori del nostro dialetto fatta consistere giustamente nel profferire quegli articoli e quelle preposizioni nel modo che più si avvicina alla lingua comune, non cessando di essere proprio al dialetto», ${ }^{20}$ mentre Achille Torelli raccomanda di scegliere, fra le varie forme di una parola dialettale, quella «che più si approssima alla lingua», rassicurando, comunque, che "[n] on si tratta di uscire dalla parlata popolare, ma di restarci». ${ }^{21} \mathrm{D}$ 'altro canto, sono proprio i riformatori ad appellarsi in modo più esplicito all'autorità di Galiani: la dura condanna che questi aveva espresso nei confronti dei classici secenteschi (includendovi gli usi grafici) perché avrebbero tradito quella tradizione colta e seria del dialetto, rivive, indipendentemente dalle motivazioni addotte dall'abate, in Imbriani e nei suoi discepoli. Naturalmente, il quadro è profondamente mutato e il discorso degli uni e degli altri, influenzato piuttosto dalle coordinate del lungo dibattito ottocentesco sulla norma linguistica dell'italiano, appare decisamente lontano da come Galiani l'aveva impostato nel suo saggio.

Le argomentazioni degli accademici, come ho accennato, sono ricalcate su quelle che aveva esibito una lunga tradizione classicistica, di cui si ribadiscono innanzitutto il principio della ineluttabile differenza fra scritto e parlato («Niuno potrà negare che ogni linguaggio scritto si differisce in alcuna cosa dal medesimo linguaggio parlato»), ${ }^{22}$ carattere riconoscibile anche nel napoletano e in tutti gli altri dialetti «che si parlano e si scrivono nella nostra carissima penisola»; ${ }^{23}$ e il principio dell'indiscussa autorità dell'uso scritto, del primato dell'Arte sulla Natura (gli scrittori classici "sollevarono il dialetto dal trivio ai portici di Chiaia», ${ }^{24}$ perché la scrittura nobilita la lingua parlata). Dal classicismo deriva pure una moderata apertura verso i neologismi, purché realizzata nel rispetto della tradizione: «L'Accademia accoglierà nuovi vocaboli, perché la scienza fa tuttogiorno nuove scoperte. Dabiturque licentia sumpta pudenter. Come nella lingua, così nel dialetto». ${ }^{25}$

Tuttavia, a differenza dei classicisti del primo Ottocento, e a prova che il romanticismo non è passato invano, il dialetto non è affatto considerato un parlare da ignoranti o da oche. Al contrario, il dialetto è rivalutato nella sua facoltà di veicolare contenuti culturali seri e razionali e non puri istinti o sentimenti primari:

20. Il dialetto napolitano si deve scrivere come si parla?, cit., p. 13.

21. A. TORELl, «La quistione dell'Ortografia», Piedigrotta, 1888 (numero unico), cit. in V. ARABIA, R. Della Campa e G. MÉry, op. cit.

22. Il dialetto napolitano si deve scrivere come si parla?, cit., p. 13.

23. Ibid., p. 8.

24. Ibid., p. 24.

25. Ibid., p. 23-24. 
Tanto una lingua quanto un dialetto sono l'espressione fonetica di un pensiero. [...] Un dialetto diventa una lingua nella lingua; mi si conceda quest'espressione, quando s'attiene all'uso, quando ha specchiati esempi, e quando ragionevolmente sa comportarsi. Avendo in non cale l'uso, non consultando i buoni autori, ed operando a capriccio la lingua diventa gergo, ed il dialetto un geroglifico. ${ }^{26}$

La considerazione del dialetto che emerge da tali affermazioni colloca il discorso su un piano antitetico a quello di Imbriani. Se questi ricacciava nella subalternità del comico la letteratura dialettale, gli accademici rivendicano l'orgoglio di una tradizione letteraria dialettale alta, degna di figurare senza complessi accanto a quella in lingua, ed avvertono il rischio che comporta la deliberata rottura nei confronti dell'eredità linguistica che essa fornisce:

Ora il dialetto napolitano ha comecchessia la sua Grammatica e parecchi vocabolari: in quella e in questi si trovano consacrate regole ed esempi da' quali non è lecito discostarsi, a meno che non si volesse avere per iscopo la totale demolizione della letteratura vernacola. ${ }^{27}$

Quando una lingua o un dialetto ha una ricca collezione di classici prosatori e poeti, ciò forma un patrimonio intangibile. E guai a chi lo tocca! Noi dobbiamo e vogliamo attenerci all'esempio di que' nostri rispettabili maestri. ${ }^{28}$

A prescindere dalle ragioni di natura più strettamente linguistica (etimologiche e grammaticali) addotte dai due schieramenti in suffragio dell'una o l'altra tesi, ragioni che vanno interpretate comunque nel quadro delle conoscenze storico-linguistiche dell'epoca, importa qui ribadire che la divergenza di fondo, difficile da conciliare, riguardava questa diversa considerazione del dialetto. Il nuovo accendersi della questione nel 1887, grazie a un'inchiesta del giornale San Carlino $^{29}$ e alla pubblicazione del volume già citato di Arabia, Della Campa e Méry, mette definitivamente a nudo questa netta divaricazione. Fra i motivi più interessanti della discussione emerge il riconoscimento, da parte dei fautori del modello classico, della presenza di varietà dialettali napoletane diastratiche e diatopiche come l'opposizione fra il parlato rozzo e il parlato civile, ${ }^{30}$ o la distinzione della pronuncia cittadina da quella dei comuni di provincia. ${ }^{31}$

26. Ibid., p. 23.

27. Ibid., p. 16.

28. Ibid., p. 24.

29. Sotto la rubrica La quistione del nostro dialetto furono pubblicati diversi interventi da gennaio fino a luglio del 1887, fra cui una polemica tra Emanuele Rocco e Michelangelo Tancredi. Quest'ultimo fu autore di un Saggio grammaticale sulla pronunzia e sull'Ortografia del dialetto napolitano, Napoli: Pierro, 1902.

30. Vi accenna Raffaele Capozzoli in un articolo sul San Carlino, IV, n. 19, cit. in V. Arabia, R. Della Campa e G. Méry, op. cit., p. 68-69. Capozzoli pubblicò una Grammatica del dialetto napoletano (Napoli: Chiurazzi, 1889), basata sulla lingua degli scrittori classici.

31. Cfr. Osservazioni del prof. Emanuele Rocco sul libro intitolato L'ortografia del dialetto napoletano, 1888. 
I riformatori, dal canto loro, negano la prima, considerando il dialetto italianizzato delle persone colte un "bastardume ridicolissimo» ${ }^{32}$ mentre, pur prendendo atto della seconda, non attribuiscono alcun primato alle forme meno evolute delle varietà rustiche. In realtà, lo sviluppo del dibattito è distorto dalla profonda diversità degli scopi. In risposta agli articoli apparsi sul San Carlino, Gaetano Amalfi, squalificando gli avversari per aver impostato male la questione, centra a pieno il motivo reale e sostanziale della discordia. Per i riformatori non può esistere analogia fra questione della lingua e questione del dialetto perché «un dialetto è tutt'altro. Perfezionato, diventa lingua, come un popolo incivilito — nello stretto senso — non è più del popolo». ${ }^{33}$ Sulla base di questa premessa teorica, espressione di un giudizio inequivoco sul ruolo assegnato al dialetto, si intende che negli obiettivi della riforma ortografica propugnata non rientra affatto la ricerca di uno standard linguistico che faccia da contrappunto alla realtà frammentaria del parlato dialettale: l'unico scopo perseguito è la definizione di un sistema di trascrizione adeguato a registrare, nella sua proteiforme realtà linguistica, la letteratura orale che ancora sopravvive. È più che esplicito Giulio Capone quando ritiene «un grave errore giudicare un dialetto e la sua grafia con criteri ricavati dalle lingue, perché i rapporti che passano tra una lingua e la sua ortografia e un dialetto e la sua trascrizione sono differentissimi». ${ }^{34}$ Se essi non avvertivano l'esigenza di uno standard era perché, secondo la loro percezione, «lo scrivere in dialetto [...] oggi, è un puro esercizio dilettantesco; ed un vano sogno l'aspirare ad una futura letteratura dialettale» e "lo scribacchiare in vernacolo ha uno scopo, molto, molto secondario». ${ }^{35}$ Simili affermazioni possono sembrare oggi sorprendenti se si pensa che l'adozione del dialetto nella letteratura postunitaria di tutta l'Italia, e di Napoli non meno che di altri centri, diventa, come scrive De Blasi, "fenomeno più vistoso di quanto non fosse stato in precedenza, quasi che giungesse una generale legittimazione per un esercizio prima praticato da pochi e solo in circostanze particolari». ${ }^{36} \mathrm{Ma}$ non stupiscono se le interpretiamo non tanto come una constatazione di fatto quanto come una dichiarazione d'intenzioni. In realtà, Emmanuele Rocco aveva capito che dietro le ragioni linguistiche della riforma di Imbriani si manifestava un atteggiamento di chiusura nei confronti della letteratura dialettale: si sottovalutava il patrimonio del passato e si guardava con diffidenza e disinteresse ai suoi possibili sviluppi presenti e futuri. In un articolo pubblicato sul San Carlino così conclude l'intervento:

Per chiarire alcune cose dette mi resta a dire che di queste novità fu caposcuola Vittorio Imbriani, forte ingegno, che sapea molte cose ma che spesso cadeva nello strano e nel paradosso. Egli nelle sue pubblicazioni, raccogliendo canti

32. V. Arabia, R. Della Campa e G. Méry, op. cit., p. 69.

33. G. Amalfi, "Come va scritto il vernacolo partenopeo?», Giambattista Basile, v, n. 6, 15 giugno 1887, p. 41.

34. G. CAPONE, Scritti inediti sui dialetti napoletano e irpino, cit., p. 25.

35. Ibid., p. 42.

36. N. DE BLASI, op. cit., p. 833. 
popolari, racconti e leggende, volle mettere in voga il dialetto parlato anziché lo scritto, rinnegando in tal modo tutta la nostra letteratura dialettale. ${ }^{37}$

Comunque sia, di fronte alla contundenza degli interventi dei demologi, il discorso di quelli che difendono a spada tratta la lingua della tradizione letteraria dialettale si rivela sul piano teorico più debole e confuso. Gli accademi$\mathrm{ci}$, pur riuscendo a controbattere pericolose generalizzazioni con intuizioni pregevoli, rifiutando per esempio l'appiattimento del dialetto sull'unico registro del parlato popolare, bastiscono le loro teorie su vecchi cliché e non si sforzano per aggiornare metodi e linguaggio. Naturalmente, anche il fronte dei riformatori presenta significative contraddizioni e ambiguità, perché raccoglie adesioni da coloro che, a differenza di Imbriani e Amalfi, non escludono né biasimano nuove attività creative, anzi mostrano un marcato interesse per la letteratura dialettale colta contemporanea. Ciò li porta ad auspicare interventi normativi, non immediatamente conciliabili con il principio di obiettività e di non interferenza che spinge i demologi nelle loro proposte di trascrizione linguistica. Per esempio, Arabia, Della Campa e Méry, nella serie di articoli raccolti nel volume già citato, dopo aver passato in rassegna le diverse convenzioni grafiche usate in passato e in presente, si soffermano sui "modernissimi», cioè su autori come Russo e Di Giacomo, rilevando ambiguità e incoerenze nelle scelte adottate nei loro scritti letterari e sostenendo, di conseguenza, la necessità di porvi rimedio. E c'è addirittura chi auspica la compilazione di una "grammatica del dialetto napoletano parlato" sulla base di un testo scritto in napoletano che dovrebbe essere redatto previamente e che dovrebbe trattare, in prosa e nel linguaggio corrente, "argomenti accessibili alle menti volgari", ritraendo "con verità la vita reale del popolo».38 Questa sorta di testo di lingua confezionato ad hoc dovrebbe servire peraltro anche a "colmare il gran vuoto che si avverte nella moderna letteratura del nostro dialetto", vale a dire l'assenza di una prosa moderna in napoletano. Per quanto eccentrico, quest'appello, lanciato da un redattore del Giambattista Basile, Ernesto Palumbo, è sintomo di un modo d'impostare il problema in cui, invece di dare per inesorabile il più o meno rapido assorbimento del dialetto nella lingua, ci si preoccupa di consolidare gli strumenti per una ripresa quantitativa e qualitativa della letteratura dialettale. L'atteggiamento di Palumbo si muove in direzione contraria a quella di Amalfi che, nella recensione al Vocabolario napoletano-italiano di Raffaele Andreoli (1888), avvertiva che bisognava cercare di "abbassare il predominio dei dialetti o lingue particolari» a favore dell'italiano e si auspicava che «de' dialetti si facesse un pochino, come del limone: spremiamo l'asprigno generoso e gittiamo via la buccia, benché questa esclusione sia difficile, finché vi sarà volgo, cioè sempre». L'asprigno generoso, cioè «la parte migliore e vitale» doveva passare «a poco a poco, nella 
lingua comune, rinsanguandola, vivificandola, accrescendole forza e leggiadria». ${ }^{39}$ D'altronde, è ben noto che, nel corso della lunga questione della lingua, i dialetti avevano subito attacchi da molteplici fronti, fino ad essere additati come uno dei maggiori intralci nel processo di unificazione linguistica. In tale contesto, si comprende che lo sforzo degli accademici fosse rivolto a mettere al riparo, attraverso la lingua della tradizione scritta, il patrimonio letterario dialettale e la sua memoria storica di fronte al processo di rapida decadenza che sembrava dipanarsi nella realtà postunitaria. Nel contestare la riforma di Imbriani, Giacomo Bugni aveva osservato a questo proposito:

Che se si dovesse avverare il manzoniano vaticino, che la lingua italiana debba prevalere, unificandosi in tutta la nostra penisola, noi del dialetto conserveremo almeno l'illustre cadavere intatto, e tale da scorgervi quale e quanto era vivente; così che non pervenga a' posteri inesplicabile come le tavole eugubine. ${ }^{40}$

A questo punto, possiamo tentare di formulare alcune conclusioni riguardo sia alla portata e alle ragioni di questi fermenti teorici intorno al napoletano sia ai fattori che li propiziarono. In primo luogo, abbiamo visto come la questione dell'ortografia del napoletano, benché sorta come un problema quasi esclusivamente tecnico di trascrizione di testi orali, si trasforma in una questione di più ampio respiro, che sconfina in aspetti sostanziali quali la ricerca di una norma linguistica e le funzioni assegnate al dialetto. In secondo luogo, dietro l'accanita difesa di un'articolazione del dialetto sui due piani dello scritto e del parlato si scorge la volontà di respingere il tentativo di riduzione del dialetto a mero registro basso destinato a scomparire, contrapponendovi una realtà in cui il dialetto mantiene vivacità e forza creativa, aspirando a essere uno strumento di usi colti e scritti in una sorta di bilinguismo letterario italiano-napoletano. In terzo luogo, l'intensa attività grammaticale e lessicografica che, almeno sul piano quantitativo, non aveva precedenti, testimonia l'interesse per una ricerca finalizzata non solo a facilitare un uso passivo del dialetto o a favorire l'apprendimento dell'italiano (come succede con la proliferazione dei dizionari bilingui), ma rivolta anche, attraverso la compilazione di grammatiche che descrivono i caratteri morfologici e sintattici del napoletano, a fornire gli strumenti per un uso attivo più consapevole e disciplinato.

Tra i fattori che condizionano e danno impulso a questo dibattito credo che ci siano almeno tre elementi importanti di cui occorre tener conto. Innanzitutto, l'eredità delle lunghe e svariate discussioni ottocentesche sulla questione della lingua che, da un lato, fornivano gli strumenti teorici e, dall'altro, avevano creato una forte sensibilità nei confronti di problemi quali la codificazione linguistica o il rapporto fra scritto e parlato. In secondo luogo, l'affermazione di indirizzi positivisti negli studi eruditi e di poetiche veriste e

39. G. AMALFI, «Un altro vocabolario napolitano», Giambattista Basile, vI, n. 8, 15 agosto 1888, p. 59.

40. Il dialetto napolitano si deve scrivere come si parla?, cit., p. 26. 
naturaliste, che aveva indotto scrittori e intellettuali a fare i conti con la realtà dialettale dei ceti popolari. Infine, le nuove prospettive sociolinguistiche, che delineavano, a breve o a lungo termine, una diffusione capillare dell'italiano in tutti i domini destinata ad alterare i tradizionali equilibri esistenti fra lingua e dialetto. Sembra quanto mai verosimile, dunque, che davanti alle spinte verso l'italianizzazione del nuovo stato unitario e davanti a una politica linguistica che scelse ben presto la via del monolinguismo manzoniano di contro alle indicazioni ascoliane che consigliavano una maggiore disponibilità nei confronti dei dialetti, si manifesti, per contraccolpo, un interesse rinnovato per la letteratura dialettale. Tale interesse contiene chiari elementi di rivendicazione, nella misura in cui risponde a un tentativo di preservare gli usi letterari alti del napoletano da una liquidazione affrettata: nella difesa della dignità linguistica del dialetto si esprime in ultima istanza la difesa della dignità della propria cultura. 